\title{
Fatores Prognósticos para o Óbito Perinatal em Gestações com Diástole Zero ou Reversa na Dopplervelocimetria das Artérias Umbilicais
}

\author{
Prognostic Parameters for Perinatal Death in Pregnancies with Absent or Reversed \\ End-Diastolic Flow Velocity in the Umbilical Arteries
}

Roseli Mieko Yamamoto, Rossana Pulcineli Vieira Francisco, Seizo Miyadahira Cátia Cristine Chuba, Marcelo Zugaib

\begin{abstract}
RESUMO
Objetivos: estudar os fatores prognósticos para o óbito perinatal em gestações com diagnóstico de diástole zero (DZ) ou reversa (DR) na dopplervelocimetria das artérias umbilicais.

Métodos: foram analisadas retrospectivamente 204 gestantes com DZ ou DR, sendo realizados os exames de cardiotocografia, perfil biofísico fetal, indice do liquido amniótico e dopplervelocimetria do ducto venoso e das artérias umbilicais, uterinas, aorta e cerebral média. Em 170 casos foi aplicado o modelo de regressão logística para determinar a variável com melhor acurácia na predição do óbito perinatal.

Resultados: a mortalidade foi de 28 casos de óbito fetal (13,7\%) e 45 de óbito pós-natal (22, 1\%). Houve correlação significativa entre os óbitos e as variáveis analisadas. A proporção de óbitos no grupo com recém-nascidos de peso inferior a $1.000 \mathrm{~g}$ foi de 74,7\% e no grupo com idade gestacional inferior a 31 semanas, de 66,3\%. Na regressão logística, o peso do recém-nascido foi a melhor variável capaz de predizer o óbito perinatal $(p<0,0001)$, permitindo a elaboração de uma curva de probabilidade de óbito de acordo com esta variável.

Conclusões: a DZ ou DR representam um grave comprometimento fetal, cujo risco para óbito perinatal está relacionado principalmente ao peso do $R N$ e à idade gestacional inferior a 31 semanas.
\end{abstract}

PALAVRAS-CHAVE: Diástole zero. Diástole reversa. Dopplervelocimetria. Artérias umbilicais. Óbito fetal.

\section{Introdução}

O fluxo sangüíneo nos vasos do cordão umbilical (artérias e veia umbilicais) depende diretamente do débito cardiaco fetal e da resistência ou complacência placentária. Do total de fluxo que flui pela aorta fetal, 50 a $60 \%$ é destinado para as artérias umbilicais que, na continuidade, participam do sistema viloso terciário que constitui uma extensa rede terminal vascular de

Setor de Vitalidade Fetal, Clínica Obstétrica do Hospital das Clínicas da Faculdade de Medicina da Universidade de São Paulo

Correspondência:

Roseli Mieko Yamamoto

Rua General Canavarro, 280 Bairro Campestre

09070-440 - Santo André - SP

Tel: (11) 4991-2481, 441-8778; FAX: (11) 441-8752

e-mail: roselinomura@uol.com.br

Home page: http://www.hcnet.usp.br/ob/ baixa resistência, local de processamento das trocas entre mãe e feto. A diástole zero (DZ) e a diástole reversa (DR) correspondem às alterações de maior gravidade observadas na dopplervelocimetria das artérias umbilicais. Apontam intenso comprometimento da função placentária que acompanha freqüentemente quadros clínicos maternos muito graves. O aumento da resistência vascular placentária, secundária ao desenvolvimento inadequado do sistema viloso terciário, e a obliteração ou redução do número das pequenas arteriolas são os fatores determinantes da redução do fluxo diastólico final da artéria umbilical ${ }^{1,2}$.

Para que ocorra a placentação normal, fenômenos vasculares obrigatórios e importantes (invasão trofoblástica) devem ocorrer ao nivel das artérias espiraladas, as responsáveis pela perfusão do espaço interviloso $\left(\right.$ EIV) ${ }^{3}$. Fisiologica- 
mente, tendo a camada fibromuscular (camada média) destruída, a resistência imposta ao fluxo sangüineo é baixa, resultando rico aporte de oxigênio no EIV, fundamental para o desenvolvimento dos vilos placentários, notadamente no terceiro trimestre, período em que o feto triplica o seu peso ${ }^{4}$. Fica claro, portanto, a estreita relação de dependência do desenvolvimento do sistema viloso terciário com a qualidade da perfusão do EIV, que por sua vez está diretamente relacionada com os fenômenos líticos proporcionados pela invasão do citotrofoblasto na camada média das artérias espiraladas.

As gestações que cursam com alterações placentárias graves são reveladas quando se diagnostica a DZ ou DR na dopplervelocimetria das artérias umbilicais. A mortalidade perinatal associada a estes diagnósticos é elevada, variando entre 15 e $60 \%$ nas maiores casuísticas referidas na literatura ${ }^{5}$. Isto faz este grupo de gestantes ser muito especial, merecedoras de tratamento diferenciado e intensivo, principalmente porque freqüentemente se relacionam a doenças maternas muito graves, atingindo o produto conceptual de forma intensa, muitas vezes em períodos críticos da gestação no limiar da viabilidade, exigindo um respaldo de cuidados neonatais intensivos e altamente especializados.

O Setor de Vitalidade Fetal da Clínica Obstétrica do Hospital das Clínicas da Faculdade de Medicina da Universidade de São Paulo (HCFMUSP) recebe contingente significativo destas doentes, principalmente por constituir serviço de nível terciário, referência para o acompanhamento de gestantes de alto risco. Em virtude disto, este estudo foi elaborado tendo por objetivo principal enfocar os fatores associados à mortalidade perinatal, aspecto de extrema relevância na decisão dos casos que evoluem claramente para o decesso do concepto.

\section{Pacientes e Métodos}

Entre fevereiro de 1992 e junho de 1999, 6.251 gestações de alto risco foram avaliadas pelo Setor de Vitalidade Fetal. Um total de 204 gestações simples em fetos estruturalmente normais, com diagnóstico de DZ ou DR à dopplervelocimetria das artérias umbilicais após a $20^{\mathrm{a}}$ semana, foram analisadas retrospectivamente consultando-se o banco de dados do Setor, complementada pela análise dos prontuários quando necessário. Este estudo foi aprovado pela Comissão de Ética para Análise de Projetos de Pesquisa da Diretoria Clínica do HC-FMUSP.
A dopplervelocimetria foi realizada por via transabdominal, utilizado-se os seguintes equipamentos de ultra-sonografia: Diasonics SPA1000 com sonda de Doppler pulsátil (1992 a 1995), ATL Ultramark 5 com Doppler colorido (1995 a 1999) e Toshiba SSA-350A com Doppler colorido (1998 a 1999). Os exames foram realizados com a paciente em posição semi-sentada. Os dados sonográficos dos vasos analisados foram obtidos com as imagens congeladas durante a inatividade fetal e em períodos de apnéia. Foram utilizados filtros de baixa freqüência ( 25 ou 50 hertz) e analisados, no mínimo, cinco sonogramas, com ondas uniformes e ângulo de incidência inferior a $60^{\circ}$, pois ângulos maiores resultam em sonogramas de amplitude baixa, dificultando sua interpretação.

$\mathrm{Na}$ dopplervelocimetria das artérias umbilicais, procura-se insonar a artéria umbilical na porção próxima à inserção placentária ${ }^{6}$. A análise neste trecho exclui a resistência decorrente do próprio cordão umbilical. A ausência de fluxo diastólico final na dopplervelocimetria das artérias umbilicais analisadas próxima à inserção placentária caracterizou o diagnóstico de $\mathrm{DZ}$, e a ocorrência de fluxo reverso neste local caracterizou a DR. Durante os primeiros quatro anos da pesquisa, o índice de pulsatilidade da artéria umbilical não era calculado, mas a partir de 1996, a avaliação dopplervelocimétrica das artérias umbilicais incluiu a análise deste índice.

Para a insonação das artérias uterinas com Doppler colorido por via transabdominal, utilizamos como referência o ponto de cruzamento destes vasos com a artéria e veia ilíaca externa. Apesar de a imagem do cruzamento entre as artérias ser um artefato devido ao plano diagonal em que se insona a pelve, é muito útil na identificação e na padronização da dopplervelocimetria destes vasos. Foi analisada a artéria correspondente ao lado da inserção placentária, sendo estudadas a relação sístole/diástole (A/B) e a presença ou não da incisura diastólica, que se caracteriza por uma ascensão discreta da velocimetria no início da diástole. Após a 26a semana de gestação a relação A/B é considerada normal até o valor de $2,6^{7}$.

O perfil hemodinâmico fetal incluiu a avaliação dos seguintes vasos da circulação fetal: aorta, artéria cerebral média e ducto venoso. A aorta fetal foi estudada em sua porção torácica descendente, facilmente identificada quando se realiza um corte longitudinal do tórax fetal. Localiza-se anteriormente e à esquerda da coluna vertebral, e as medidas são realizadas na sua porção intratorácica, acima do músculo diafragmático. 
Para a localização do melhor ponto de avaliação da artéria cerebral média a seguinte técnica foi utilizada: inicialmente é visibilizado um corte transverso do polo cefálico fetal no nível onde realiza-se a medida do diâmetro biparietal, na altura dos tálamos; em seguida, a porção anterior do transdutor deve ser deslocada obliquamente, em direção à base do crânio, até um nível um pouco acima do osso esfenóide. Neste ponto a artéria é facilmente identificada como um ramo maior do polígono de Willis que se dirige anterolateralmente em direção à fissura de Sylvius. Deve-se evitar a compressão do abdome materno, uma vez que a compressão do polo cefálico fetal pode levar a alterações nos exames realizados nas artérias intracranianas.

O ducto venoso é identificado efetuandose um corte oblíquo do abdome fetal, na altura da inserção do cordão umbilical. A porção intraabdominal da veia umbilical é facilmente visibilizada, identificando-se a sua bifurcação em seio portal e ducto venoso. Na porção inicial do ducto ocorre um turbilhonamento do sangue, provocando um efeito de mistura de cores (efeito "aliasing"), onde pode-se obter o sonograma característico deste vaso.

O sonograma da aorta foi classificado como alterado quando o resultado do indice de pulsatilidade se encontrava acima do percentil 95 da curva de normalidade de Arduini e Rizzo de 1990, para a idade gestacional correspondente $^{8}$. Na avaliação da artéria cerebral média, para o diagnóstico de centralização da circulação fetal, os resultados do índice de pulsatilidade foram classificados como alterados quando se encontravam abaixo do percentil 5 da curva de normalidade de Arduini e Rizzo ${ }^{8}$, em idade gestacional correspondente. O sonograma do ducto venoso foi analisado utilizando-se do índice de pulsatilidade para veias (S-a/média), que expressa a relação da diferença entre a velocidade de pico sistólica (S) e a velocidade mínima na contração atrial (a), com a velocidade média. O seu resultado foi classificado como anormal quando foi superior ao percentil 95 da curva de normalidade de Hecher et al. ${ }^{9}$, na idade gestacional correspondente. Neste estudo, analisamos os resultados da última avaliação do perfil hemodinâmico fetal realizado antes do parto ou do óbito fetal.

A cardiotocografia (CTG) foi realizada por um período mínimo de 20 minutos e classificada como normal na presença de acelerações transitórias e/ou quando o feto se apresentava reativo após o estímulo sônico (aceleração da freqüência cardiaca fetal - FCF - de $20 \mathrm{bpm}$ por três minutos). Foi considerado alterado quando o padrão cardiotocográfico foi caracterizado como ina- tivo, de acordo com o índice cardiotocométrico de Zugaib e Behle ${ }^{10}$. Foi também verificada a presença de desacelerações da FCF. Os DIPs do tipo II foram caracterizados como desacelerações tardias da FCF, com decalagem superior a 18 segundos em relação à contração uterina. Também foram consideradas desacelerações a ocorrência de DIPs do tipo umbilical e as bradicardias.

No perfil biofisico fetal (PBF) foram estudados os seguintes parâmetros: cardiotocografia, movimentos respiratórios fetais, movimentos corpóreos fetais, tônus fetal e índice do líquido amniótico. A avaliação do volume de líquido amniótico foi realizada subjetivamente no primeiro ano do estudo e depois pelo cálculo do índice de líquido amniótico (ILA), de acordo com a metodologia proposta por Phelan et al. ${ }^{11}$. O oligoidrâmnio foi diagnosticado quando o valor do ILA se encontrava inferior ou igual a $5,0 \mathrm{~cm}$ e o oligoidrâmnio grave quando inferior ou igual a 3,0 $\mathrm{cm}$. Foram analisados os resultados obtidos na última avaliação da vitalidade fetal realizada antes do parto ou do óbito fetal.

A realização das provas de maturidade fetal obedeceram ao protocolo assistencial de conduta na $\mathrm{DZ}$ ou $\mathrm{DR}^{12}$. O líquido amniótico foi analisado pelo teste de Clements (TC) em 3 tubos e/ ou pelo índice citolipídico, que consiste na contagem de células orangiófilas $(\mathrm{CO})$ coradas pelo azul de Nilo a $0,1 \%$. O feto foi classificado em: maduro, quando o TC foi positivo nos três tubos e/ou contagem de $\mathrm{CO}$ superior ou igual a $10 \%$; intermediário quando o TC foi positivo até o segundo tubo e/ou $\mathrm{CO}$ entre 6 a $9 \%$, e imaturo, quando o TC foi negativo nos três tubos ou positivo apenas no primeiro, com $\mathrm{CO}$ inferior a $5 \%$. Foram analisados somente os resultados do último exame realizado nas pacientes, antes do parto.

$\mathrm{Na}$ avaliação dos resultados perinatais, as seguintes variáveis foram estudadas: idade gestacional no diagnóstico da $\mathrm{DZ}$ ou $\mathrm{DR}$, intervalo entre o diagnóstico de $\mathrm{DZ}$ ou $\mathrm{DR}$ e o parto, $\mathrm{pH}$ da artéria umbilical no nascimento, índices de Apgar de 1ㅇ, 5ㅇ e $10^{\circ}$ minutos, idade gestacional no nascimento, peso do recém-nascido (RN), incidência de RN pequenos para a idade gestacional (PIG), necessidade de entubação orotraqueal do RN na sala de parto, internação do RN em UTI neonatal e óbitos perinatais. Os RN foram classificados como PIG quando apresentaram peso inferior ao percentil 10 para a idade gestacional, de acordo com as curvas de normalidade desenvolvida pelo Setor de Neonatologia deste hospital. Todos os RN foram acompanhados no berçário, sendo que a análise da mortalidade incluiu os óbitos fetais e os que ocorreram duran- 
te a internação do RN no berçário.

A idade gestacional foi calculada a partir da data da última menstruação (DUM) quando esta foi compativel com exame ultra-sonográfico realizado até a 20a semana. Quando a paciente não sabia referir a DUM ou na discordância com o exame ultra-sonográfico, a datação da gestação foi realizada com base na primeira ultrasonografia realizada. Quando não foi possivel calcular-se a idade gestacional com estes critérios, utilizou-se do método de Capurro somático para a estimativa da idade gestacional.

Na Clínica Obstétrica do Hospital das Clínicas da FMUSP ${ }^{12}$, para gestantes com DZ ou DR, preconiza-se a orientação resumida a seguir:

1) internação da paciente após o diagnóstico, para repouso e avaliação rigorosa da vitalidade fetal e realização de ultra-sonografia morfológica. $\mathrm{Na}$ presença de anomalias fetais, a conduta estará vinculada ao diagnóstico;

2) entre a $20^{\underline{a}}$ e a $28^{\mathrm{a}}$ semana de idade gestacional, a dopplervelocimetria e o ILA são realizados 2 a 3 vezes por semana, sendo que a partir de 26 semanas realizamos também a CTG e o PBF. A resolução da gestação é considerada possivel nos casos em que a idade gestacional for superior a 26 semanas e o peso fetal estimado superior a $500 \mathrm{~g}$.

3) a partir da 28 a semana de gestação, a avaliação da vitalidade fetal é de fundamental importância. Quando esta se apresentar alterada, caracterizada pela ocorrência de desacelerações tardias na CTG ou PBF inferior a 6 ou ILA inferior a $3,0 \mathrm{~cm}$, é indicada a resolução da gestação. Em situações de vitalidade fetal preservada, é indicada a avaliação da maturidade fetal. Quando as provas indicarem maturidade plena ou intermediária, optase pela resolução da gestação. Nos fetos imaturos, preconizamos o repouso da paciente e a vigilância fetal intensiva, por meio da realização diária da cardiotocografia, por no mínimo 30 minutos, acompanhado da avaliação do PBF e do ILA. O perfil hemodinâmico fetal é realizado a cada 24 a 72 horas e a maturidade é reavaliada em 7 a 14 dias. A conduta resolutiva é adotada em doenças maternas graves, de dificil controle clínico, ou após a 34a semana de gestação.

Com o objetivo de estudar os fatores prognósticos para o óbito perinatal, procuramos analisar o maior número de variáveis disponiveis, principalmente visando obter dados que possam ser úteis na orientação destas gestantes quanto à sobrevida do concepto. Deste modo, foram constituídos dois grupos: o de RN vivos, que constituem os casos em que o RN recebeu alta do berçário em boas condições, e o de óbitos, que incluem as gestações que evoluíram para óbito fetal e aquelas cujos RN evoluíram para óbito durante a internação no berçário, independente do número de dias que transcorreu do nascimento ao óbito. Em estudos anteriores realizados neste Setor, identificamos como parâmetros de alto risco para mortalidade perinatal o peso do RN inferior a $1.000 \mathrm{~g}^{13} \mathrm{e}$ a idade gestacional ao nascimento inferior a 31 semanas. Na dopplervelocimetria da artéria umbilical, foi utilizado o limite de 2,85 para o índice de pulsatilidade, pois este valor corresponde à mediana dos dados obtidos nesta amostra. No ducto venoso, valores superiores a 1,0 para o índice de pulsatilidade estão relacionados à acidose fetal ${ }^{14}$.

Para a identificação dos fatores prognósticos de maior capacidade de predição para o óbito, foram selecionados 170 casos completos para a análise multivariada por meio do modelo de regressão logística, utilizando-se das seguintes variáveis para a análise: idade gestacional no diagnóstico da DZ ou DR; PBF inferior ou igual a 4; ILA inferior ou igual a $5,0 \mathrm{~cm}$, cardiotocografia inativa, intervalo entre a $\mathrm{DZ}$ ou $\mathrm{DR}$ e o parto (dias), idade gestacional no nascimento, presença de desacelerações, presença de DIP II e peso do RN (gramas).

Para a comparação entre os grupos de estudo, utilizou-se do teste de $\chi^{2}$ para as variáveis qualitativas e do teste $t$ de Student para as quantitativas. Adotou-se como nível de significância o valor $0,05(\alpha=5 \%)$. Com isso, niveis descritivos (p) inferiores a esse valor foram considerados significantes $(p<0,05)$. Nas correlações significativas, foram calculadas a sensibilidade, especificidade e razão de chances (odds ratio) com intervalos de confiança de $95 \%$ para cada variável. As variáveis que apresentaram significância estatística na análise univariada foram selecionadas para análise multivariada com o ajuste do modelo de regressão logística, com procedimento stepwise de seleção de variáveis, afim de identificar a variável com maior acurácia para predição do óbito perinatal.

\section{Resultados}

No periodo analisado, a incidência de $\mathrm{DZ}$ ou DR nas gestações de alto risco avaliadas pelo Setor de Vitalidade Fetal da Clínica Obstétrica do HC-FMUSP foi de $3,3 \%$. Os resultados das 204 gestações estudadas encontram-se nas tabelas a seguir. A idade média da população total 
foi de 29,4 anos com desvio padrão de 6,1 anos, não sendo evidenciadas diferenças estatisticamente significativas entre os grupos analisados. O mesmo ocorreu em relação ao número de gestações e à paridade das pacientes, como pode ser observado na Tabela 1. A idade gestacional no diagnóstico da DZ ou DR, a idade gestacional no parto e o peso do RN apresentaram resultados significativamente inferiores no grupo que evoluiu com óbito. Não houve diferença em relação ao intervalo entre o diagnóstico e o parto.

Tabela 1 - Principais características das gestações com diástole zero (DZ) ou diástole reversa (DR) nas artérias umbilicais, de acordo com a ocorrência ou não do óbito perinatal: médias e desvios-padrão (DP). IG, Idade gestacional.

\begin{tabular}{|c|c|c|c|}
\hline Características & $\begin{array}{c}\text { RN vivos }(n=131) \\
\text { média } \pm \mathrm{DP}\end{array}$ & $\begin{array}{c}\text { Óbitos }(n=73) \\
\text { média } \pm \text { DP }\end{array}$ & $\mathrm{p}$ \\
\hline Idade (anos) & $29,6 \pm 6,4$ & $29,0 \pm 5,5$ & NS \\
\hline Número de gestações & $3,0 \pm 2,0$ & $2,6 \pm 1,9$ & NS \\
\hline Paridade (número) & $1,4 \pm 1,6$ & $1,1 \pm 1,3$ & NS \\
\hline IG no diagnóstico da DZ/DR (semanas) & $31,2 \pm 3,1$ & $26,2 \pm 3,5$ & $<0,0001$ \\
\hline Intervalo entre o diagnóstico e o parto (dias) & $6,6 \pm 14,3$ & $10,4 \pm 13,0$ & NS \\
\hline IG no parto (semanas) & $32,1 \pm 2,4$ & $27,8 \pm 2,8$ & $<0,0001$ \\
\hline Peso do RN (gramas) & $1333,7 \pm 390,5$ & $758,4 \pm 242,2$ & $<0,001$ \\
\hline
\end{tabular}

A mortalidade geral foi de $35,8 \%$, com 28 casos de óbito fetal $(13,7 \%)$ e 45 de óbitos no berçário $(22,1 \%)$. No grupo de 157 gestações com DZ, a mortalidade perinatal foi de $27,4 \%$ (43 casos), e nas 47 gestações com DR a mortalidade foi de $63,8 \%$ (30 casos). Nas Tabelas 2 e 3 podemos observar a sobrevida, a mortalidade fetal e neonatal e a mortalidade cumulativa de acordo com as faixas de peso do RN e com a idade ges- tacional no nascimento. Podemos observar que a mortalidade perinatal diminui progressivamente conforme aumenta a idade gestacional no nascimento e o peso do RN. A sobrevida dos RN com peso superior a 1.400 gramas é de praticamente $100 \%$, o mesmo ocorrendo após a 34a semana de gestação. A mortalidade é muito elevada para fetos com peso inferior a 800 gramas e abaixo da 28a semana de gestação.

Tabela 2 - Sobrevida, óbito neonatal, fetal e mortalidade cumulativa nas gestações com diástole zero ou diástole reversa nas artérias umbilicais de acordo com o peso do recém nascido.

\begin{tabular}{ccccccccccc}
\hline Peso do RN (g) & Total & \multicolumn{2}{c}{ Vivos } & \multicolumn{3}{c}{ Óbito neonatal } & \multicolumn{3}{c}{ Óbito fetal } & \multicolumn{3}{c}{ Mortalidade cumulativa } \\
\hline & & $\mathbf{n}$ & $(\%)$ & $\mathbf{n}$ & $(\%)$ & $\mathbf{n}$ & $(\%)$ & $\mathbf{n}$ & total & $(\%)$ \\
$270-500$ & 10 & & - & 2 & $(20,0)$ & 8 & $(80,0)$ & 10 & 10 & $(100,0)$ \\
$501-600$ & 6 & & - & 3 & $(50,0)$ & 3 & $(50,0)$ & 16 & 16 & $(100,0)$ \\
$601-700$ & 21 & 3 & $(14,3)$ & 10 & $(47,6)$ & 8 & $(38,1)$ & 34 & 37 & $(91,9)$ \\
$701-800$ & 18 & 2 & $(11,1)$ & 11 & $(61,1)$ & 5 & $(27,8)$ & 50 & 55 & $(90,9)$ \\
$801-900$ & 18 & 10 & $(55,5)$ & 8 & $(44,4)$ & & - & 58 & 73 & $(79,5)$ \\
$901-1.000$ & 14 & 9 & $(64,3)$ & 4 & $(28,6)$ & 1 & $(7,1)$ & 63 & 87 & $(72,4)$ \\
$1.001-1.100$ & 18 & 14 & $(77,8)$ & 3 & $(16,7)$ & 1 & $(55,5)$ & 67 & 105 & $(63,8)$ \\
$1.101-1.200$ & 27 & 24 & $(88,9)$ & 2 & $(7,4)$ & 1 & $(3,7)$ & 70 & 132 & $(53,0)$ \\
$1.201-1.300$ & 13 & 12 & $(92,3)$ & 1 & $(7,7)$ & & - & 71 & 145 & $(49,0)$ \\
$1.301-1.400$ & 10 & 9 & $(90,0)$ & & - & 1 & $(10,0)$ & 72 & 155 & $(46,4)$ \\
$1.401-1.500$ & 11 & 11 & $(100,0)$ & & - & & - & 72 & 166 & $(43,4)$ \\
$1.501-1.600$ & 8 & 8 & $(100,0)$ & & - & & - & 72 & 174 & $(41,4)$ \\
$1.601-1.700$ & 7 & 6 & $(85,7)$ & 1 & $(14,3)$ & & - & 73 & 181 & $(40,3)$ \\
$1.701-1.800$ & 5 & 5 & $(100,0)$ & & - & & - & 73 & 186 & $(39,3)$ \\
$1.801-1.900$ & 6 & 6 & $(100,0)$ & & - & & - & 73 & 192 & $(38,0)$ \\
$1.901-2.000$ & 5 & 5 & $(100,0)$ & & - & & - & 73 & 197 & $(37,1)$ \\
$>2.000$ & 7 & 7 & $(100,0)$ & & - & & - & 73 & 204 & $(35,8)$ \\
\hline
\end{tabular}


Tabela 3 -Sobrevida, óbito neonatal, fetal e mortalidade cumulativas nas gestações com diástole zero ou diástole reversa nas artérias umbilicais de acordo com a idade gestacional (IG) no nascimento.

\begin{tabular}{|c|c|c|c|c|c|c|c|c|c|c|}
\hline \multirow[t]{2}{*}{ IG (sem) } & \multirow[t]{2}{*}{ Total } & \multicolumn{2}{|c|}{ Vivos } & \multicolumn{2}{|c|}{ Óbito neonatal } & \multicolumn{2}{|c|}{ Óbito fetal } & \multicolumn{3}{|c|}{ Mortalidade cumulativa } \\
\hline & & $\mathrm{n}$ & $(\%)$ & $\mathrm{n}$ & $(\%)$ & $\mathrm{n}$ & $(\%)$ & $\mathrm{n}$ & total & $(\%)$ \\
\hline$\leq 25$ & 12 & & - & 1 & $(8,3)$ & 11 & $(91,7)$ & 12 & 12 & $(100,0)$ \\
\hline 26 & 9 & 1 & $(11,1)$ & 2 & $(22,2)$ & 6 & $(66,7)$ & 20 & 21 & $(95,2)$ \\
\hline 27 & 12 & 1 & $(8,3)$ & 10 & $(83,3)$ & 1 & $(8,3)$ & 31 & 33 & $(93,9)$ \\
\hline 28 & 23 & 8 & $(34,8)$ & 10 & $(43,5)$ & 5 & $(21,7)$ & 46 & 56 & $(82,1)$ \\
\hline 29 & 18 & 10 & $(55,5)$ & 6 & $(33,3)$ & 2 & $(11,1)$ & 54 & 74 & $(73,0)$ \\
\hline 30 & 21 & 12 & $(57,1)$ & 8 & $(38,1)$ & 1 & $(4,8)$ & 63 & 95 & $(66,3)$ \\
\hline 31 & 22 & 18 & $(81,8)$ & 3 & $(13,6)$ & 1 & $(4,5)$ & 67 & 117 & $(57,3)$ \\
\hline 32 & 24 & 21 & $(87,5)$ & 3 & $(12,5)$ & & - & 70 & 141 & $(49,6)$ \\
\hline 33 & 24 & 22 & $(91,7)$ & 1 & $(4,2)$ & 1 & $(4,2)$ & 72 & 165 & $(43,6)$ \\
\hline 34 & 18 & 18 & (100) & & - & & - & 72 & 183 & $(39,3)$ \\
\hline 35 & 13 & 12 & $(92,3)$ & 1 & $(7,7)$ & & - & 73 & 196 & $(37,3)$ \\
\hline$\geq 36$ & 8 & 8 & $(100)$ & & - & & - & 73 & 204 & $(35,8)$ \\
\hline
\end{tabular}

Em relação às intercorrências clínicas e obstétricas associadas ao diagnóstico de DZ ou DR, pudemos observar que as sindromes hipertensivas foram as mais freqüentes, ocorrendo em 161 casos $(78,9 \%)$. Deste total de pacientes hipertensas, a distribuição de acordo com o tipo de hipertensão foi a seguinte: 75 casos de hipertensão arterial crônica $(46,6 \%), 48$ casos de pré-eclâmpsia $(29,8 \%), 28$ casos de toxemia superajuntada $(17,4 \%), 6$ casos de eclâmpsia $(3,7 \%)$ e 4 casos de sindrome HELLP $(2,5 \%)$. A segunda intercorrên- cia mais freqüente foi o CIUR em 35 casos $(21,7 \%)$, seguido pelo diabetes em 18 casos $(11,2 \%)$ e as cardiopatias em 16 casos $(9,9 \%)$.

$\mathrm{Na}$ avaliação da vitalidade fetal (Tabela 4), podemos observar uma relação significativa entre as alterações da cardiotocografia, o oligoidrâmnio (ILA $\leq 5,0 \mathrm{~cm}$ ) e o perfil biofísico fetal alterado $(\mathrm{PBF} \leq 4)$ com o óbito perinatal. Na análise do traçado cardiotocográfico, tanto o padrão inativo como a presença de desacelerações se relacionaram com os óbitos.

Tabela 4 - Distribuição dos resultados referentes à idade gestacional (IG) no parto, peso do recém-nascido, cardiotocografia, perfil biofísico fetal, índice do líquido amniótico e maturidade fetal, de acordo coma ocorrência ou não do óbito perinatal nas gestações com diástole zero ou diástole reversa nas artérias umbilicais.

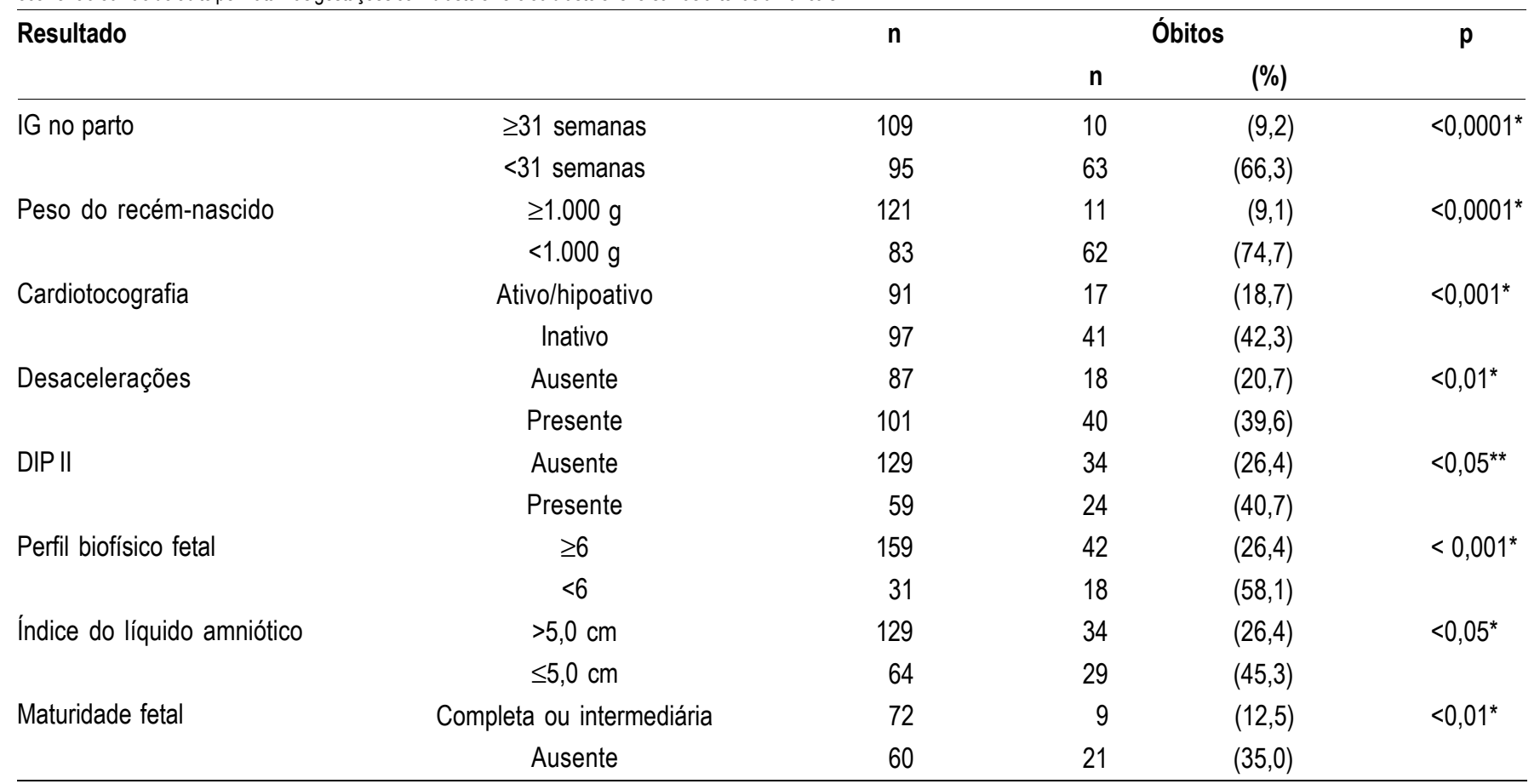

${ }^{*}$ Teste do $\chi^{2}$

** Teste do $\chi^{2}$ de Mantel-Haenszel 
Somente em 124 dos casos avaliados neste estudo foi possivel realizar a gasometria. Observamos uma elevada incidência de acidose no nascimento, diagnosticada pela medida do $\mathrm{pH}$ do sangue da artéria umbilical inferior a 7,20 (65 casos). Dentre estes casos, 18 evoluíram para óbito perinatal $(27,7 \%)$, e entre os 59 casos com $\mathrm{pH}$ normal a proporção de óbitos foi de $23,7 \%$ (14 casos), não sendo observada diferença significativa entre os grupos.

Ao estudarmos a maturidade fetal pela análise do líquido amniótico em 132 casos (Tabela 4), observamos que no grupo com imaturidade fetal $(n=60)$ houve $35 \%$ de óbito perinatal, com diferença significativa quando comparado com a mortalidade perinatal dos fetos com maturidade completa ou intermediária.

Em relação à dopplervelocimetria das ar- térias umbilicais, a ocorrência de DR apresentou-se significativamente relacionada aos óbitos perinatais (Tabela 5). Em apenas 64 casos foi calculado o índice de pulsatilidade deste vaso, que, analisado de forma contínua ou de acordo com o limite de superior ou igual a 2,85 , relacionou-se aos óbitos. As alterações na artéria uterina se correlacionaram significativamente com os óbitos quando apresentavam relação A/B superior a 2,6, o mesmo ocorrendo em relação à presença da incisura. A Tabela 5 demonstra ainda os resultados do perfil hemodinâmico fetal. As alterações no ducto venoso se correlacionaram positivamente com os óbitos. Não foram observadas diferenças estatisticamente significativas em relação à análise da aorta fetal e da artéria cerebral média, pois pudemos observar alta incidência de alterações em ambos os grupos.

Tabela 5 - Distribuição dos resultados da dopplervelocimetria obstétrica de acordo com a ocorrência ou não do óbito perinatal em gestações com diástole zero ou diástole reversa nas artérias umbilicais.

\begin{tabular}{|c|c|c|c|c|c|}
\hline \multirow[t]{2}{*}{ Resultado } & & \multirow[t]{2}{*}{$\mathrm{n}$} & \multicolumn{2}{|c|}{ Óbitos } & \multirow[t]{2}{*}{$p$} \\
\hline & & & n & $(\%)$ & \\
\hline \multirow[t]{2}{*}{ Artéria umbilical } & Diástole zero & 157 & 43 & $(27,4)$ & $<0,0000^{*}$ \\
\hline & Diástole reversa & 47 & 30 & $(63,8)$ & \\
\hline \multirow[t]{2}{*}{ Índice de pulsatilidade da artéria umbilical } & $<2,85$ & 32 & 5 & $(15,6)$ & $<0,0000^{*}$ \\
\hline & $\geq 2,85$ & 32 & 22 & $(68,7)$ & \\
\hline Artéria uterina & $A / B>2,6$ & 143 & 63 & $(44,1)$ & \\
\hline \multirow[t]{2}{*}{ Incisura na artéria uterina } & Ausente & 29 & 3 & $(10,3)$ & $<0,01^{*}$ \\
\hline & Presente & 166 & 68 & $(40,9)$ & \\
\hline Ducto venoso & Normal & 22 & 2 & $(9,1)$ & $<0,01^{*}$ \\
\hline \multirow[t]{2}{*}{ Artéria cerebral média } & Normal & 27 & 5 & $(18,5)$ & NS \\
\hline & Anormal $(\mathrm{IP} \leq \mathrm{p} 5)$ & 142 & 53 & $(37,3)$ & \\
\hline \multirow[t]{2}{*}{ Aorta } & Normal & 15 & 4 & $(26,7)$ & NS \\
\hline & Anormal (IP $\geq p 95)$ & 46 & 21 & $(45,6)$ & \\
\hline
\end{tabular}

* Teste do $\chi^{2}$

Na Figura 1 estão demonstrados os resultados neonatais na população estudada. Somente os casos de recém-nascidos vivos estão incluídos nesta análise, apresentando correlação significativa entre o índice de Apgar de $1^{\circ}$ e $5^{\circ} \mathrm{mi}-$ nuto inferior a 7 , entubação do RN na sala de parto e internação em UTI neonatal com o óbito do RN.

De acordo com a análise univariada das variáveis estudadas, foram calculadas a sensibilidade, especificidade e razão de chances (odds ratio - OD) para o óbito perinatal, estando estes resultados demonstrados na Tabela 6. As variáveis que apresentaram melhores valores de sensibilidade e especificidade foram o peso do RN inferior a $1.000 \mathrm{~g}$ e a idade gestacional inferior a 31 semanas.

$\mathrm{Na}$ análise multivariada pelo modelo de regressão logística, a variável que mostrou maior acurácia para predizer o óbito foi o peso do RN ( $p<0,0001)$, possibilitando a elaboração da curva de probabilidade de acordo com o seu resultado, conforme demonstrado na Figura 2. 


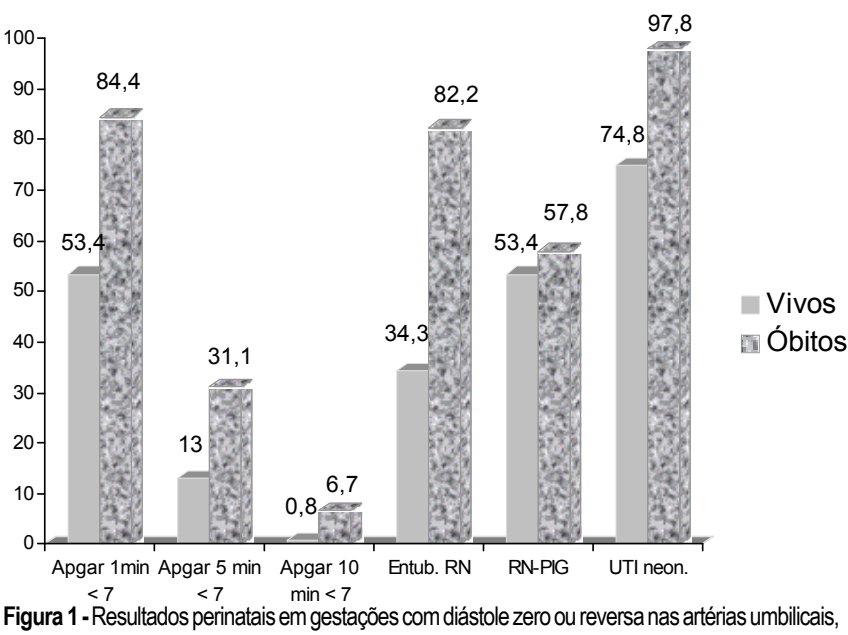
conforme a ocorrência ou não de óbito neonatal.

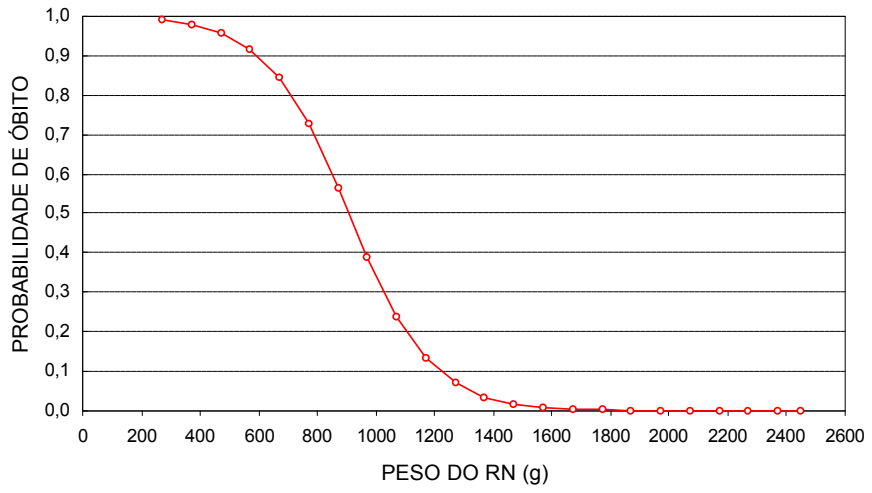

Figura 2- Curva de probabilidade de óbito perinatal de acordo com o peso do recém-nascido (RN) em gestações com diástole zero ou reversa nas artérias umbilicais.

Tabela 6 - Valores da sensibilidade (S), especificidade (E), (odds ratio, OR) e intervalo de confiança (IC)a 95\% para as variáveis com diferença estatisticamente significativa para a ocorrência do óbito perinatal em gestações com diástole zero ou diástole reversa nas artérias umbilicais.

\begin{tabular}{lllll}
\hline Variável & $\mathbf{S ~ ( \% )}$ & $\mathbf{E ~ ( \% )}$ & OR & IC a 95\% \\
\hline Peso do recém nascido <1.000 g & 84,9 & 83,9 & 29,5 & $12,5-71,2$ \\
Idade gestacional $<31$ sem & 86,3 & 75,6 & 19,5 & $8,5-46,0$ \\
Diástole reversa & 41,1 & 87,0 & 4,7 & $2,2-9,9$ \\
IP da artéria umbilical $\geq 2,85$ & 81,5 & 73,0 & 11,9 & $3,1-49,4$ \\
Ducto venoso alterado & 92,9 & 48,8 & 12,4 & $2,5-17,2$ \\
IP do ducto venoso $\geq 1,0$ & 57,1 & 78,0 & 4,7 & $1,5-15,7$ \\
Doppler da artéria uterina A/B >2,6 & 88,7 & 35,5 & 4,3 & $1,8-10,8$ \\
Incisura na artéria uterina & 95,8 & 21,0 & 6,0 & $1,7-32,0$ \\
Líquido amniótico imaturo & 70,0 & 61,8 & 3,8 & $1,4-10,0$ \\
Cardiotocografia inativa & 70,7 & 56,9 & 3,2 & $1,6-6,5$ \\
Cardiotocografia c/ desacelerações & 68,9 & 53,1 & 2,5 & $1,2-5,1$ \\
Cardiotocografia c/ DIP II & 41,4 & 73,1 & 1,9 & $0,9-3,9$ \\
Perfil biofísico fetal $\leq 4$ & 30,0 & 90,0 & 3,9 & $1,6-9,2$ \\
Indice do líquido amniótico $\leq 5,0 \mathrm{~cm}$ & 46,0 & 73,1 & 2,3 & $1,2-4,6$ \\
Índice de Apgar do $1^{\circ}$ min $<7^{*}$ & 84,4 & 46,6 & 4,7 & $1,9-13,4$ \\
Índice de Apgar do $5^{\circ}$ min $<7^{*}$ & 31,1 & 87,7 & 3,0 & $1,2-7,4$ \\
\hline
\end{tabular}

${ }^{*}$ Nascidos vivos

\section{Discussão}

A incidência da DZ ou DR na população geral é baixa, sendo que Johnstone et al. ${ }^{15}$ não descrevem nenhum caso num grupo de 160 gestantes sem complicações. Nas pacientes que apresentam gravidez de alto risco, a incidência de DZ ou DR varia entre 1 e $34 \%^{3}$, de acordo com os diferentes critérios de inclusão utilizados. No nosso estudo, a incidência de 3,3\% está de acordo com os dados referidos na literatu$\mathrm{ra}^{15,16}$.

Apesar dos elevados índices de mortalida- de fetal e neonatal associados ao diagnóstico de $\mathrm{DZ}$ e DR, os estudos mais recentes com casuísticas maiores demonstram que a indicação da resolução da gestação deve ser criteriosa, ponderando-se sempre os riscos da prematuridade extrema e as seqüelas da asfixia intrauterina, e também a eficiência da unidade de terapia intensiva neonatal local ${ }^{17,18}$.

Deste modo, é fundamental se determinar quais os fatores prognósticos relacionados ao óbito perinatal, estabelecendo-se seus valores de predição para melhor interpretação dos casos e para orientar a conduta obstétrica. Karsdorp et al. ${ }^{19}$, em estudo multicêntrico envol- 
vendo nove centros europeus, analisaram os resultados perinatais em 178 gestantes com DZ e 67 com DR, e observaram mortalidade perinatal nos casos com DZ de $41 \%$ e com DR, de $75 \%$. No presente estudo, a mortalidade geral na população analisada foi de $35,8 \%$, sendo na DZ de $27,4 \%$ e na DR de $63,8 \%$. Pacientes com fluxo reverso no sonograma apresentam mortalidade perinatal muito elevada, freqüentemente superior à da $\mathrm{DZ}^{20}$. Brar e Platt ${ }^{21}$ foram os primeiros a ressaltar a gravidade da DR na artéria umbilical. Do mesmo modo, observamos uma elevada mortalidade nos casos de diástole reversa da artéria umbilical. Este diagnóstico está associado a elevados valores do índice de pulsatilidade da artéria umbilical que, por sua vez, apresentou também correlação significativa com a mortalidade perinatal, com boa sensibilidade e especificidade para este resultado, indicando ser esta uma variável importante na assistência das gestantes com o diagnóstico de DZ ou DR.

A grande maioria das pacientes com $\mathrm{DZ}$ ou DR apresentou como intercorrência clínica mais freqüente as sindromes hipertensivas, concordando com outros estudos da literatura ${ }^{13,22,23}$. Observamos também uma elevada incidência de RN pequenos para a idade gestacional (cerca de $55 \%$ ), demonstrando o elevado grau de insuficiência placentária a que estes fetos são submetidos cronicamente, comprometendo a sua nutrição e oxigenação. A mortalidade perinatal elevada evidencia o alto risco destes RN, necessitando, quase sempre, de cuidados intensivos e assistência neonatal especializada na sala de parto. A entubação do RN é um procedimento freqüente durante a assistência nos primeiros minutos de vida, principalmente no grupo de maior risco para mortalidade neonatal. Os indices de Apgar no primeiro e quinto minutos de vida também são parâmetros prognósticos para a mortalidade neonatal.

Determinar o momento oportuno para a resolução da gestação é o maior problema após o diagnóstico da DZ ou DR. Esta decisão é particularmente crítica, no nosso Serviço, no final do segundo e início do terceiro trimestres da gestação. As decisões clínicas geralmente se baseiam no nosso protocolo assistencial, porém, condutas personalizadas são freqüentes, baseadas em todas as informações obtidas a respeito da condição fetal e do quadro clínico materno. O peso estimado e a idade gestacional influenciam diretamente na conduta a ser adotada. Estas duas variáveis demonstraram ser as mais importantes a delinear o prognóstico do RN, que também está diretamente vinculado à eficiência da assistência intensiva neonatal. No nosso serviço, a mortalidade nos casos com idade gestacional inferior a 28 semanas e para $\mathrm{RN}$ com peso inferior a $800 \mathrm{~g}$ é muito elevada, restringindo a conduta obstétrica a uma atitude de observação nestas situações. Porém, quando a morte do concepto é iminente, mesmo cientes da alta mortalidade neonatal, optamos pela resolução da gestação, após discussão com a gestante e seu companheiro dos riscos e beneficios do procedimento. Enquanto as condições hemodinâmicas e biofisicas fetais permitirem o prolongamento da gestação, procuramos sempre aguardar um momento mais oportuno.

A detecção precoce das alterações hemodinâmicas da circulação fetal é, portanto, de fundamental importância, pois elas refletem os mecanismos de adaptação do concepto em face do comprometimento da sua oxigenação ${ }^{24}$. Durante o processo de hipoxia fetal, ocorrem alterações na circulação fetal com redistribuição do débito cardíaco, fornecendo maior fluxo sangüíneo para órgãos nobres (território cerebral e coronariano) em detrimento de outros órgãos como os rins, pulmões e trato gastrointestinal. Este processo, também conhecido como centralização da circulação fetal ou brain-sparing effect, pode ser diagnosticado por meio do perfil hemodinâmico fetal, observando-se aumento da velocidade diastólica no fluxo sangüíneo das artérias cerebrais, conseqüente à vasodilatação deste território. Clinicamente este dado corresponde à primeira alteração provocada pela hipoxemia fetal. Enquanto a centralização mantém a capacidade de compensar a oxigenação do sistema nervoso central, as atividades biofísicas permanecerão normais. Na hipoxia grave, com perda dos mecanismos compensatórios, os sinais de sofrimento fetal agudo são evidentes, com alterações importantes na cardiotocografia e no perfil biofisico fetal.

A avaliação do fluxo sangüíneo na aorta fetal permite estudar a resistência vascular periférica fetal, quando os mecanismos de redistribuição da circulação fetal promovem vasoconstrição das regiões periféricas. $\mathrm{Na}$ hipoxemia fetal, inicialmente ocorre um aumento do fluxo no ducto venoso. Este vaso consiste numa intercomunicação da circulação fetal que liga a veia umbilical à veia cava inferior, promovendo o transporte de maior parte do sangue oxigenado para o coração fetal. Com o agravamento da hipoxia, ocorre o aumento da resistência vascular pela vasoconstricção periférica. Observa-se um aumento da resistência na aorta fetal e uma elevação na pressão diastólica final nas câmaras direitas do coração fetal, que aumentam o fluxo retrógrado da veia cava inferior, 
durante a contração atrial. Este processo se reflete no ducto venoso com redução do fluxo neste vaso, podendo, em casos graves, tornar-se reverso na contração atrial.

O Setor de Vitalidade Fetal, ao estudar a circulação fetal nos casos de DZ ou DR, procura estabelecer o estágio hemodinâmico da resposta fetal à hipoxia. O fenômeno da centralização da circulação fetal é freqüente nos referidos casos, por ser uma resposta precoce no mecanismo adaptativo fetal. A avaliação dopplervelocimétrica do ducto venoso vem apresentando bons resultados, pois se relaciona à acidose fetal ${ }^{12}$ nestes casos, representando o comprometimento importante da oxigenação do feto. Evidenciase, assim, a necessidade de avaliação da hemodinâmica fetal em gestações com DZ ou DR, auxiliando na determinação do momento mais oportuno para a resolução destas gestações.

Baseando-se em estudos realizados neste Serviço $^{25}$, em face da presença de maturidade fetal intermediária ou completa indica-se a interrupção da gestação, pois as lesões decorrentes da hipoxemia fetal podem ser mais intensas que as decorrentes da prematuridade. A amniocentese para avaliação do líquido amniótico é um procedimento importante na determinação da conduta em gestações viáveis. A partir de 28 semanas, em estudos realizados no Setor de Vitalidade Fetal, é possivel diagnosticar maturidade fetal e, também, a presença de mecônio no liquido amniótico.

Existem várias limitações metodológicas que restringem as análises neste estudo retrospectivo. A incidência de DZ ou DR é relativamente baixa e a coleta destes 204 casos se fez em sete anos, período longo em que mudanças nos equipamentos de ultra-sonografia ocorreram, porém, o protocolo assistencial vem se mantendo relativamente estável neste período. É muito discutível a adoção de qualquer tipo de randomização prospectiva para o seguimento e conduta nestas pacientes, principalmente em face da extrema gravidade dos casos e do elevado risco para a perda do concepto.

Portanto, nos casos de DZ ou DR, é imprescindivel rigorosa monitorização da vitalidade fetal por meio de todos os exames disponiveis, visando evitar o diagnóstico tardio de sofrimento fetal. Em alguns casos o controle do bem-estar fetal permite que fetos extremamente prematuros e até mesmo inviáveis possam ser acompanhados até por períodos de semanas, permitindo o nascimento de recém-nascido viável e em melhores condições de maturidade. Neste aspecto, o perfil hemodinâmico fetal representa um papel fundamental, pois pode nos fornecer valiosas infor- mações com respeito ao prognóstico da gestação, auxiliando na determinação do momento mais oportuno de resolução das gestações com DZ ou DR.

Conclui-se, então, com bases nestes dados, que o risco para o óbito perinatal está relacionado principalmente ao peso do $\mathrm{RN}$ e à idade gestacional ao nascimento, principalmente para aqueles com peso inferior a $1.000 \mathrm{~g}$ ou abaixo da 31a semana de gestação. Utilizando-se do modelo de regressão logística, este estudo permitiu a elaboração de uma curva de probabilidade para o óbito perinatal de acordo com o peso do RN, facilitando a interpretação do risco de óbito de acordo com o peso estimado do feto, nas gestações que cursam com DZ ou DR na dopplervelocimetria das artérias umbilicais.

\section{SUMMARY}

Purpose: to study the prognostic parameters for perinatal death in pregnancies with absent or reversed end-diastolic flow velocity on umbilical artery dopplervelocimetry.

Methods: two hundred and four pregnancies were retrospectively reviewed. The methods used were cardiotocography, fetal biophysical profile, amniotic fluid index and dopplervelocimetry of ductus venosus, fetal aorta, middle cerebral artery, umbilical arteries and uterine artery. The logistic regression model was applied to one hundred and seventy cases in order to determine the most accurate variable for predicting perinatal death. Results: the mortality rates were: 28 cases of intrauterine fetal death (13.7\%) and 45 neonatal deaths (22.1\%). A statistically significant correlation was found between death and the studied variables. The perinatal death rate in the group with birth weight below 1,000 g was $74.7 \%$, and in the group with gestational age at delivery below 31 weeks it was $66.3 \%$. By logistic regression, birth weight was the most accurate variable for predicting perinatal death, and a probability curve for death according to this variable was obtained.

Conclusions: absent or reversed end-diastolic flow velocity in the umbilical arteries is a severe fetal condition, where the risk of perinatal death is mainly related to birth weight and a gestational age at delivery below 31 weeks.

KEY WORDS: Absent end-diastolic flow velocity. Reversed end-diastolic flow velocity. Dopplervelocimetry. Umbilical arteries. Fetal death. 


\section{Referências}

1. Miyadahira S, Yamamoto RM, Francisco RPV, Steinman DS, Schultz R, Zugaib M. Placental characteristics in pregnancies with absent or reversed end-diastolic velocity flow in the umbilical artery. Ultrasound Obstet Gynecol 1998; 12 Suppl 1:159.

2. Salafia CM, Pezzulo JC, Minior VK, Divon MY. Placental pathology of absent and reversed enddiastolic flow in growth-restricted fetuses. Obstet Gynecol 1997; 90:830-6.

3. Pijnenborg R, Dixon G, Robertson WB, Brosens I. Trophoblastic invasion of human decidua from 8 to 18 weeks of pregnancy. Placenta $1980 ; 1: 3-19$.

4. Pritchard JA, MacDonald PC, Gant NF. The morphologic and functional development of the fetus. In: Pritchard JA, MacDonald PC, Gant NF, editors. Williams Obstetrics. 17th ed. Newark: Appleton-Century-Crofts; 1985. p.139-80.

5. Farine D, Kelly EN, Ryan G, Morrow RJ, Knox Ritchie JW. Absent and reversed umbilical artery enddiastolic velocity. In: Copel JA, Reed KL, editors. Doppler ultrasound in obstetrics and gynecology. 1st ed. New York: Raven Press; 1995. p.187-97.

6. Abramowicz JS, Warsof SL, Arrington J, Levy DL. Doppler analysis of the umbilical artery. The importance of choosing the placental end of the cord. J Ultrasound Med 1989; 8:219-21.

7. Fleischer A, Schulman H, Farmakides G, et al. Uterine artery doppler velocimetry in pregnant women with hypertension. Am J Obstet Gynecol 1986; 154:806-13.

8. Arduini D, Rizzo G. Normal values of pulsatility index from fetal vessels: a cross sectional study on 1556 healthy fetuses. J Perinat Med 1990; 18:165-72.

9. Hecher K, Campbell S, Snijders R, Nicolaides K. Reference ranges for fetal venous and atrioventricular blood flow parameters. Ultrasound Obstet Gynecol 1994; 4:381-90.

10.Zugaib M, Behle I. Monitoração fetal eletrônica. $1^{\mathrm{a}}$ ed. São Paulo: Roca; 1981. p.55-78.

11.Phelan JP, Smith CV, Broussard P, Small M. Amniotic fluid volume assessment with the fourquadrant technique at 36-42 weeks' gestation. J Reprod Med 1987; 32:540-2.

12.Yamamoto RM, Miyadahira S, Francisco RPV, Steinman DS, Aquino MA, Zugaib M. Conduta obstétrica na diástole zero ou reversa à dopplervelocimetria das artérias umbilicais. Rev Ginecol Obstet 1999; 10:35-9.

13.Steinman DS. Gestantes hipertensas com diástole zero ou reversa no exame dopplervelocimétrico da artéria umbilical: análise da vitalidade fetal e dos resultados perinatais [dissertação]. São Paulo: Universidade de São Paulo; 1998.

14.Francisco RPV. Predição da acidose no nascimento em gestações com diástole zero ou reversa à dopplervelocimetria das artérias umbilicais [dissertação]. São Paulo: Universidade de São Paulo; 1998.

15.Johnstone FD, Haddad NG, Hoskins P, McDicken W, Chambers S, Muir B. Umbilical artery Doppler flow velocity waveform: the outcome of pregnancies with absent end-diastolic flow. Eur J Obstet Gynecol Reprod Biol 1988; 28:171-8.

16.Forouzan I. Absence of end-diastolic flow velocity in the umbilical artery: a review. Obstet Gynecol Surv 1995; 50:219-27.

17.Kurkinen-Räty M, Kivelä A, Jouppila P. The clinical significance of an absent end-diastolic velocity in the umbilical artery detected before the 34th week of pregnancy. Acta Obstet Gynecol Scand 1997; 76:398-404.

18.Weiss E, Ulrich S, Berle P. Condition at birth of infants with previously absent or reverse umbilical artery end-diastolic flow velocities. Arch Gynecol Obstet 1992; 252:37-43.

19.Karsdorp VH, van Vugt JM, van Geijn HP, et al. Clinical significance of absent or reversed end diastolic velocity waveforms in umbilical artery. Lancet 1994; 344:1664-8.

20.Franzin CMMO, Silva JLP, Mezzalira R, Surita F, Marussi EF. Componente distólico ausente na artéria umbilical: análise de 30 casos. Rev Bras Ginecol Obstet 1996; 18:491-4.

21.Brar HS, Platt LD. Reverse end diastolic flow velocity on umbilical artery velocimetry in high risk pregnancies: an ominous finding with adverse pregnancy outcome. Am J Obstet Gynecol 1988; 159:559-61.

22.Rochelson B, Schulman H, Farmakides G, et al. The significance of absent end-diastolic velocity in umbilical artery velocity waveforms. Am J Obstet Gynecol 1987; 156:1213-8.

23.Arduini D, Rizzo G, Romanini C. The development of abnormal heart rate patterns after absent enddiastolic velocity in umbilical artery: analysis of risk factors. Am J Obstet Gynecol 1993; 168:4350 .

24.Weiner Z, Farmakides G, Schulman H, Penny B. Central and peripheral hemodynamic changes in fetuses with absent end-diastolic velocity in umbilical artery: correlation with computerized fetal heart rate pattern. Am J Obstet Gynecol 1994; 170:509-15.

25.Yamamoto RM, Francisco RPV, Miyadahira S, Steinman DS, Zugaib M. Amniotic fluid analysis in pregnancies with absent or reversed enddiastolic velocity flow in the umbilical artery. Ultrasound Obstet Gynecol 1998; 12 Suppl 1:162. 